\title{
Outcomes of total knee replacement with the use of a NexGen MIS Tibial Component (Mini-keel) : a systematic review
}

\author{
Naoki Nakano, Kiyonori Mizuno, Koji Takayama, Shinya Hayashi, Ryosuke Kuroda, Tomoyuki Matsumoto \\ From the Department of Orthopaedic Surgery, Kobe University Graduate School of Medicine
}

\begin{abstract}
NexGen MIS Tibial Component (Mini-keel) is a tibial component specially developed for minimally invasive surgery in total knee replacement (TKR), and the size limitations of its design and the modular system could affect tibial fixation strength, however, this has not been precisely evaluated thus far. This study aimed to systematically review the literature describing the outcome following TKR with the use of a Mini-keel. Electronic searches of databases were undertaken in July 2019 by two experienced orthopaedic surgeons according to the PRISMA guidelines for literature describing the outcomes of TKR with the use of a Mini-keel. Quality of studies was assessed using the Joanna Briggs Institute Critical Appraisal Checklist. The initial search found a total of 623 studies from all the databases. Seven studies met all the inclusion criteria and were eligible for critical appraisal and quality assessment. In total, 2,198 cases were included in the systematic review.

Thirty-five revision cases due to aseptic loosening were found from the systematic review. Two studies were negative about using a Mini-keel and three studies were positive about it, while the other two studies did not judge the quality of a Mini-keel. There have been conflicting conclusions among studies for the use of a Mini-keel.

There remains a paucity of prospective cohort studies between TKRs with the use of a Mini-keel and those with the use of a conventional implant, which makes it difficult to determine the usefulness and reliability of this implant.
\end{abstract}

No benefits or funds were received in support of this study. None of the authors have a conflict of interest.
Keywords : knee ; total knee replacement ; minimally invasive surgery; NexGen MIS Tibial Component; systematic review.

\section{INTRODUCTION}

Total knee replacement (TKR) is a successful and technically well-established procedure in the treatment of end-stage degenerative joint disease or deformity of the knee with a rate of survival of more than $90 \%$ after 15 years of follow-up (1-3). It is becoming increasingly popular among people of working age as there is a trend among working

Naoki Nakano ${ }^{1,2}, \mathrm{MD}, \mathrm{PhD}$

Kiyonori Mizuno ${ }^{2}, \mathrm{MD}, \mathrm{PhD}$

- Koji Takayama ${ }^{1,2}, \mathrm{MD}, \mathrm{PhD}$

- Shinya Hayashi ${ }^{1}, \mathrm{MD}, \mathrm{PhD}$

- Ryosuke Kuroda ${ }^{1}, \mathrm{MD}, \mathrm{PhD}$

- Tomoyuki Matsumoto ${ }^{1}, \mathrm{MD}, \mathrm{PhD}$

${ }^{1}$ Department of Orthopaedic Surgery, Kobe University Graduate School of Medicine, 7-5-1 Kusunoki-cho, Chuoku, Kobe 650-0017, Japan.

${ }^{2}$ Department of Orthopaedic Surgery, Anshin Hospital, 1-412, Minatojimaminami-machi, Chuo-ku, Kobe 650-0047, Japan.

Correspondence : Naoki Nakano Department of Orthopaedic Surgery, Kobe University Graduate School of Medicine, 7-5-1 Kusunoki-cho, Chuo-ku, Kobe 650-0017, Japan Phone : +81 (0) 78382 5985. Fax : +81 (0) 783516944.

Email : gnaokix1981@gmail.com

- 2021, Acta Orthopædica Belgica. 
adults choosing to delay retirement (4), and a less invasive approach have become more demanding expectations (5). At its birth in 2003, minimally invasive surgery (MIS) in TKR (MIS-TKR) started with the purpose of reducing the size of the incisions and the damages to the soft tissues (6-8). MIS-TKR is believed to be helpful for short skin incision, smaller soft tissue dissection, reduced postoperative pain, decreased use of painkillers, shorter recovery and hospitalisation periods, fast recovery of the range of motion as well as the muscular strength, reduced need for postoperative manipulation, lower cost, and fewer postoperative aesthetics (9-11). However, in MIS-TKR, the risks of malpositioning of the prosthesis and wound problems with inadequate visualisation of the operative field could be increased because compared with conventional techniques, it involves a smaller exposed surgical site (12). Moreover, with this technique, the tibial implant must be inserted without tibiofemoral dislocation, which cannot be easily conducted with the use of standard components. To overcome these problems, a lot of efforts have been made to reduce the size of the instruments to make them fit through smaller incisions, and also to improve the components in order to make the implanting technique easier through small approaches and limited exposures (13). The NexGen MIS Tibial Component (Mini-keel) (Zimmer Inc., Warsaw, IN, USA) (Fig. 1) is one of the inventions that can be inserted into the knee without anterior dislocation of the knee joint due to the presence of a minimised keel (14). It is made of two parts : the plate with a keel underneath and a modular stem ("drop-down" stem extension). The size limitations of its design and the modular system could affect tibial fixation strength, however, this has not been precisely evaluated in a clinical setting. Currently, there is a lack of evidence in the literature that provides knee surgeons with evidence-based recommendations on the use of a Mini-keel for TKR, and no systematic review has been published in this arena so far. The aim of this systematic review, therefore, was to provide the reader with the related outcomes for MIS-TKR with the use of a Mini-keel based on the best available evidence.

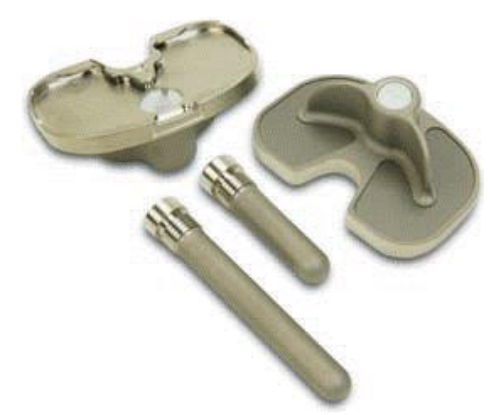

Fig. 1. - NexGen MIS Tibial Component (Mini-keel); Zimmer Biomet Inc., Warsaw, IN, USA.

\section{MATERIAL AND METHODS}

The PICOS tool was adopted to formulate the research question and modified since no comparators were sought in this study (15). Two reviewers searched the online databases (MEDLINE, PubMed, Cochrane Central Register of Controlled Trials and Cochrane Database of Systematic Reviews) for literature describing the outcomes of TKR with the use of a Mini-keel. The Preferred Reporting Items for Systematic Reviews and Meta-Analyses (PRISMA) guidelines were used for designing this study (16). Database search was conducted on 1st, July 2019 and retrieved articles from the databases since inception to the search date. The electronic search citation algorithm used was : ((TKR OR TKA OR (total knee replacement) OR (total knee arthroplasty)) AND (low-profile OR modular OR mini-keel* OR short-keel* OR MIS OR (minimally invasive surgery)) AND tibia* (Title/Abstract)). The search also included the yet to be printed search results. Results were pooled and exported to EndnoteX6 software (Thomson Reuters, USA) and duplicates were removed electronically and manually. The two reviewers independently reviewed all the titles and abstracts. The remaining search results were divided equally between the two reviewers and reviewed in duplicate applying the inclusion and exclusion criteria. Any discrepancies at the title and abstract stage as well as the full-text stage were resolved by consensus between the two reviewers and the third more senior author. This process led to $100 \%$ agreement between the authors.

Levels 1, 2, 3, 4, and 5 evidence (according to the Oxford Centre for Evidence-Based Medicine) 
Table I. - Inclusion and exclusion criteria applied to articles identified in the literature

\begin{tabular}{|l|}
\hline Inclusion criteria \\
\hline 1. All levels of evidence \\
2. Written in the English language \\
3. Studies on humans \\
4. Studies reporting the outcome of total knee replacement using NexGen MIS Tibial Component (Mini-keel) \\
5. Operative procedure consisted solely of total knee replacement \\
6. Total knee replacement irrespective of any pathology \\
\hline Exclusion criteria \\
\hline 1. Studies describing trial protocols without any results \\
2. Total knee replacement not using the mini-keel modular tibial implant \\
3. Total knee replacement without describing details of used implants \\
4. Revision total knee replacement \\
5. Unicompartmental knee replacement \\
6. Patellofemoral knee replacement \\
7. Animal studies \\
8. Basic science studies (e.g. cadaveric studies) \\
9. Diagnostic studies without any description of treatments \\
10. Technical notes without any results \\
11. Reviews, systematic reviews \\
\hline
\end{tabular}

English-language studies were eligible for inclusion in the systematic review. We excluded duplicate subject publications within separate unique studies. Both electronically published articles and print journal were included for this review. Clinical outcome studies, prospective case series, retrospective case series and case reports that described the outcomes of TKR with the use of a Mini-keel irrespective of any pathology were included. Studies describing the results of unicompartmental knee replacement, patellofemoral knee replacement, and revision TKR were excluded. Studies on animal models and basic science studies (e.g. cadaveric studies), studies describing trial protocols without any results, diagnostic studies without any description of treatments, technical notes without any results, and review articles were also excluded. The detailed inclusion and exclusion criteria are shown in Table I.

Both the reviewers independently extracted the relevant study data from the final pool of included articles and recorded this data on a spreadsheet designed a priori in Microsoft Excel 2013 (Microsoft Corporation, Redmond, Washington, USA). Participant-specific demographics extracted from each study included the number of cases and patients, gender distribution, mean age with range (years), original disease, surgical approach, mean operation time, details of stem extension, type of femoral component, cementing technique, final outcome, and specific notes (if any). Study-specific demographics extracted from each study included the level of evidence according to the simplified evidence level table from the Centre for EvidenceBased Medicine, Oxford, country where the study was conducted and the year of publication. Studies were then analysed and assessed using the Joanna Briggs Institute Critical Appraisal Checklist (JBICAC) for case series and case-control studies. A scoring system was implemented based on the findings from the studies. JBICAC scores the answers to its questions as yes, no, unclear or not applicable. We then allocated numbers to each answer where domains answering with yes gets 2 points, unclear gets 1 and no gets 0 . A scoring of 20 indicated the maximum points of both case series and case-control studies. The extracted data was then analysed using Microsoft Excel 2013. Statistical analyses in this study focused on descriptive statistics.

\section{RESULTS}

The initial search found a total of 623 studies from all the databases. The search process led to 100\% agreement among authors. Duplicates found 
were 76 articles and were removed. A total of 547 articles were then identified for title screening. Three hundred and sixty-seven articles were excluded based on the inclusion criteria leaving 180 articles for abstract screening. Eighty-seven articles were then excluded and 93 articles were included for full-text review. Eventually, seven studies (four case-control studies and three case series) met all the inclusion criteria and were eligible for critical appraisal, quality assessment and included in the study. One study was a comparative study between Mini-keel and standard tibial implant (Kajetanek et al (17)), and others were studies describing the outcomes of Mini-keel only (The other three casecontrol studies focused on other factors (type of femoral component (18), obesity (19) and varus deformity (20)) and did not compare Mini-keel with standard components.). The oldest study included in this review was published in 2011. In total, 2,198 TKRs in 1,922 patients were included in the systematic review. Of the participants, $20.6 \%$ were male and $79.4 \%$ were female (data availability: $67.3 \%$ ). Mean age of the participants was 68.3 years (range, 48 to 88 years). Of the seven studies finally included in this systematic review, two studies (Foran (21) and Kajetanek (17)) were negative about using a Mini-keel and three studies (Benazzo (14), Yoo (22) and Yang (18)) were positive about it, while the other two studies (Yoo(19) and Yoo (20)) did not judge the quality of a Mini-keel. Thirty-five revision cases due to aseptic loosening were found from the systematic review. Other than revisions, five complications (three cases of rupture of the medial collateral ligament, one case of detachment of the cortex from the medial femoral condyle, and one case of periprosthetic tibial fracture) were reported.

The flow chart of the literature search algorithm is shown in Fig. 2. Due to the lack of homogeneity in studies, a meta-analysis was deemed unsuitable for this study. Detailed data extracted from all the studies is shown in Table II. The outcomes of quality assessment using JBICAC were shown in Table III.

\section{DISCUSSION}

MIS-TKR, which has become popular in the past 15 years thanks to advances in surgical techniques and instrumentation, involves minimum damage to the suprapatellar pouch without patellar eversion and tibiofemoral joint dislocation, which aimed to minimise damage and tension in the soft tissue. Using downsized guiding tools and cutting zigs have made reducing the length of skin incision and damage to the underlying structures possible in MIS-TKR. Reported advantages of MIS-TKR such as the faster recovery of muscle strength, less blood loss, and shortened hospital stay have encouraged knee surgeons to use this technique for patients (23),

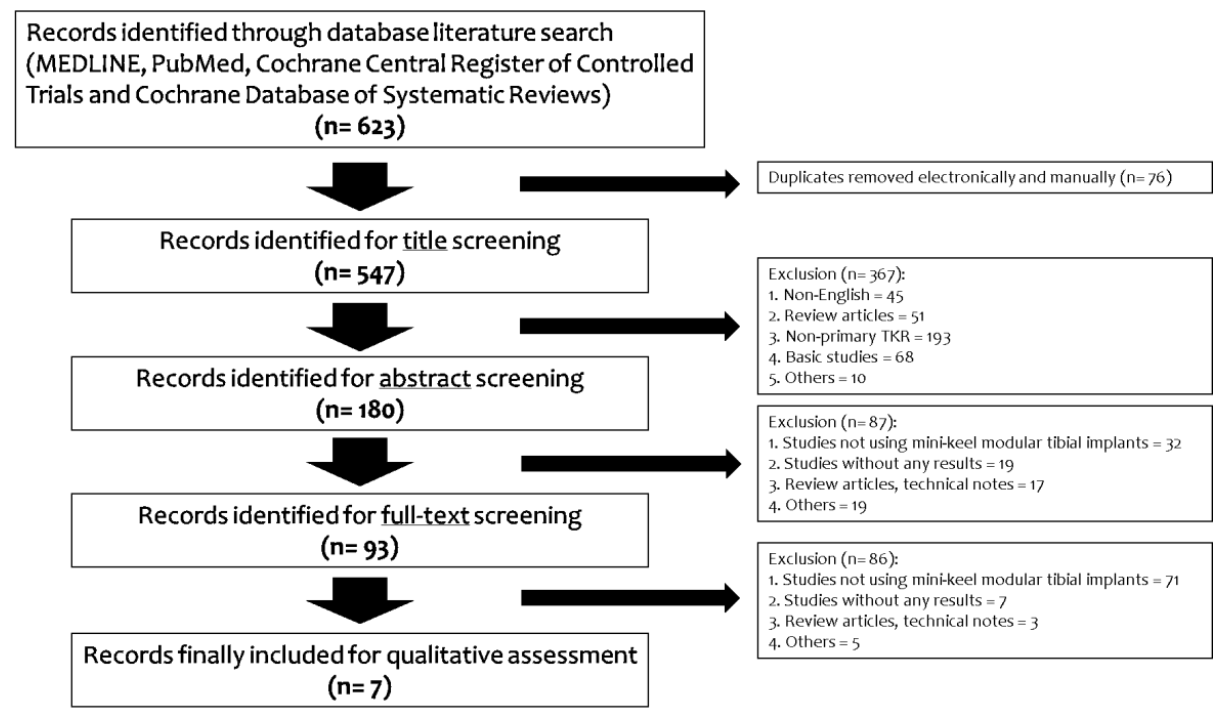

Fig. 2. - PRISMA flowchart for results of the literature database search. 
while it still requires an experienced surgeon and takes time to obtain the necessary surgical skills.

While MIS-TKR can be performed through a small incision, it can be challenging if the field of view is extremely limited $(12,13)$. The tibial resection surface should be sufficiently anteriorly dislocated with the knee flexed when conventional tibial components are used. If the length of the incision is small, complications may occur by excessive tension on the adjacent tissue. Mini-keel is designed to facilitate insertion and manipulation of prostheses in the smaller surgical fields to overcome these disadvantages. It is made of titanium alloy and composed of a tibial plate with a minimised keel and a $45 \mathrm{~mm}$ - or $75 \mathrm{~mm}$-long modular drop-down stem extension. Thanks to its short keel length (range, 17 to $19 \mathrm{~mm}$ ), it prevents tension on the surrounding tissue and additional soft tissue resection when it is inserted. In addition, it provides a $5.20 \%$ wider contact surface than normal tibial component, which contributes to the stability of the knee. Although there have been a small number of clinical studies of Mini-keel, there has been no systematic review in this arena thus far.

This systematic review finally found seven studies, two of which were negative about using a Mini-keel and three of which were positive about it, while the other two focused on patient's factors and did not comment on the quality of a Mini-keel. Both of the two negative studies opposed to the use of a Mini-keel because of the high revision rate due to aseptic loosening and no studies reported inferior clinical outcomes of this implant such as HSS score, Knee Society Score and knee range of motion. In the result of the quality assessment of the seven included studies using JBICAC tool, the study of Benazzo et $\mathrm{al}^{14}$ and that of Yang et al. (18) showed higher quality than other studies in the category of case series and case-control studies, respectively, and both of the two studies are positive about the use of a Mini-keel. Potential advantages of this implant, i.e. faster recovery following TKR or less postoperative pain thanks to smaller approaches and limited exposures, have not been researched or judged in any studies.

One of the negative studies (Foran et $\mathrm{al}^{21}$ ) did not use drop-down stem extensions and it might result in a high revision rate due to aseptic loosening. In an attempt to obtain further stability by increasing the interface between the implant and the tibia, additional use of a drop-down stem extension has been strongly recommended by the manufacturer since June 2010. In fact, the study by Kajetanek et a ${ }^{17}$ showed higher revision rate of the cases without a stem extension (33.3\%) compared with those with a $45 \mathrm{~mm}$ stem extension (4.9\%) and those with a $75 \mathrm{~mm}$ stem extension (16.7\%), while no statistical differences among them were described. Foran et al also mentioned that another possibility of their poor results could be unfavorable design features of a Mini-keel, and it is possible that the geometry of a Mini-keel may lead to unfavorable stress shielding in vivo and weakening of the underlying bone, which increases susceptibility to bone loss as well as osteolysis (21). Kajetanek et al mentioned that the design of this implant involves an additional interface between the plate and the keel, which also could be a source of loosening (17).

The quality of the cement technique may also have contributed to the result in each study. An optimal cement amount and distribution with good pressurisation is very important for secure fixation and long-term durability of TKRs, and even more critical for tibial components with short keels like a Mini-keel. The manufacturer released a document in July 2009 detailing proper cement technique for a Mini-keel and described that "The MIS Tibial Component is designed for cemented use. The instrumentation prepares a cement mantle around the keel with a line-to-line fit at the ends of the fins. For this reason, cement must be applied within the tibial medullary canal as well on the proximal tibial surface, under the tibial plate and around the keel". Benazzo et al. (14) emphasised the importance of cementing techniques through analysing cement volume, distribution, and penetration from followup radiographs, while they did not document the correlation of radiolucency. It was reported that a safe cement penetration should be between 3 and $4 \mathrm{~mm}$, and the pattern of distribution of the cement is coherent with the design of the implant and the cementing technique used (24).

From this systematic review, 5 complications (3 cases of rupture of the medial collateral ligament, 
Table II. - Details of 7 studies included in the systematic review

\begin{tabular}{|c|c|c|c|c|c|c|c|c|c|c|}
\hline \multirow[t]{2}{*}{ Author } & \multirow[t]{2}{*}{ Year } & \multirow[t]{2}{*}{ Country } & \multirow[t]{2}{*}{ Type of the study } & \multicolumn{3}{|c|}{ Number of patients } & \multirow[t]{2}{*}{ Number of TKRs } & \multirow[t]{2}{*}{ Follow-up period } & \multicolumn{2}{|c|}{ Age (years) } \\
\hline & & & & Male & Female & Total & & & Mean & Range \\
\hline Foran [21] & 2011 & USA & Case series & NA & NA & 460 & 529 & NA & 61 & $56-73$ \\
\hline Benazzo [14] & 2012 & Italy & Case series & 139 & 36 & 175 & 200 & 3 years (mean) & 69 & $52-88$ \\
\hline Yoo [22] & 2014 & Korea & Case series & NA & NA & 168 & 168 & 6 years and 1 month (mean) & 68.8 & $53-80$ \\
\hline Kajetanek [17] & 2016 & France & Case control & 47 & 165 & 212 & 212 & 59.4 months (mean) & 73.2 & $47.4-88.5$ \\
\hline Yoo [20] & 2016 & Korea & Case control & 30 & 415 & 445 & 627 & 5 months (mean) & 71.8 & NA \\
\hline Yoo [19] & 2018 & Korea & Case control & 26 & 345 & 371 & 371 & Minimum 5 years & 69.3 & $53-80$ \\
\hline Yang [18] & 2018 & Taiwan & Case control & 25 & 66 & 91 & 91 & Minimum 5 years & 69.4 & $48-85$ \\
\hline Author & \multicolumn{10}{|c|}{ Cementing } \\
\hline Foran [21] & \multirow{2}{*}{\multicolumn{10}{|c|}{$\begin{array}{l}\text { Immediately before cementing, the bony surfaces were cleaned with high-pressure, high-volume pulsatile lavage and then dried. Cement was then finger-packed to } \\
\text { enhance cement penetration. Palacos (Zimmer) antibiotic- } \\
\text { impregnated cement was used. } \\
\text { Components were cemented with antibiotized Simplex (Stryker Howmedica) after pulsed lavage, without pressurization of the cement. The cementing technique } \\
\text { consists of dropping the cement in the dough phase into the drilled hole, putting cement underneath the tibial plate, then impacting it. }\end{array}$}} \\
\hline Benazzo $[14]$ & & & & & & & & & & \\
\hline Yoo [22] & \multicolumn{10}{|c|}{ Cemented (Details are not described) } \\
\hline Kajetanek [17] & \multicolumn{10}{|c|}{$\begin{array}{l}\text { Components were cemented in one stage with high viscosity Palacos bone cement (Heraeus), including the keel. The cancellous femoral and tibial surfaces were } \\
\text { washed under pressure with saline solution then dried. The cement for the tibial component was applied under the base plate with a cement mantle around the tibial } \\
\text { keel. }\end{array}$} \\
\hline Yoo [20] & \multicolumn{10}{|c|}{ Not described (TKRs in Figures seem to be cemented) } \\
\hline Yoo [19] & \multicolumn{10}{|c|}{ Not described (TKRs in Figures seem to be cemented) } \\
\hline Yang [18] & \multicolumn{10}{|c|}{ The fully cemented technique with pressurization was used. } \\
\hline
\end{tabular}

TKR, total knee replacement ; OA, osteoarthritis ; RA, rheumatoid arthritis ; ON, osteonecrosis ; BMI, body mass index. 
Table II. - Details of 7 studies included in the systematic review

\begin{tabular}{|c|c|c|c|c|c|}
\hline $\begin{array}{l}\text { BMI TKR, total knee replacement; OA, osteoarthritis; RA, rheuma- } \\
\text { toid arthritis; ON, osteonecrosis; BMI, body mass index. }\left(\mathrm{kg} / \mathrm{m}^{2}\right)\end{array}$ & $\begin{array}{l}\text { Mean Operation } \\
\text { time }\end{array}$ & $\begin{array}{l}\text { Drop-down stem } \\
\text { (length) }\end{array}$ & $\begin{array}{c}\text { Femoral } \\
\text { component }\end{array}$ & Disease & Approach \\
\hline NA & NA & Not used & $\mathrm{CR}$ & $\mathrm{OA}$ & $\begin{array}{l}\text { Subvastus approach } \\
\text { or midvastus approach }\end{array}$ \\
\hline NA & $90 \mathrm{~min}$ & $45 \mathrm{~mm}$ & PS (LPS-Flex) & $\begin{array}{l}\text { OA: } 178 \text { cases, Post- } \\
\text { trauma: } 22 \text { cases }\end{array}$ & $\begin{array}{l}\text { Mini-midvastus } \\
\text { approach: } 172 \text { cases, } \\
\text { mini-trivector approach: } \\
35 \text { cases }\end{array}$ \\
\hline $27 \pm 3.7($ mean \pm SD $)$ & $87.5 \mathrm{~min}$ & $45 \mathrm{~mm}$ & PS (LPS-Flex) & $\begin{array}{l}\text { OA: } 165 \text { cases, RA: } 2 \\
\text { cases, ON: } 1 \text { case }\end{array}$ & $\begin{array}{l}\text { Mini-midvastus } \\
\text { approach }\end{array}$ \\
\hline 28.73 (mean) & NA & $\begin{array}{l}\text { Not used: } 3 \text { cases, } \\
45 \mathrm{~mm}: 203 \text { cases, } \\
70 \mathrm{~mm}: 6 \text { cases }\end{array}$ & PS (LPS-Flex) & $\begin{array}{l}\text { OA: } 206 \text { cases, } \\
\text { Others: } 6 \text { cases }\end{array}$ & $\begin{array}{l}\text { Medial trans- } \\
\text { quadricipital parapatellar } \\
\text { approach }\end{array}$ \\
\hline 27.2 (mean) & $85.3 \mathrm{~min}$ & $45 \mathrm{~mm}$ & PS (LPS-Flex) & $\begin{array}{l}\text { OA: } 622 \text { cases, RA: } 3 \\
\text { cases, ON: } 2 \text { cases }\end{array}$ & $\begin{array}{l}\text { Mini-midvastus } \\
\text { approach }\end{array}$ \\
\hline 27.1 (mean) & $86.7 \mathrm{~min}$ & NA & PS (LPS-Flex) & $\begin{array}{l}\text { OA: } 367 \text { cases, RA: } \\
4 \text { cases }\end{array}$ & $\begin{array}{l}\text { Mini-midvastus } \\
\text { approach }\end{array}$ \\
\hline 27.5 (mean) & $164.3 \mathrm{~min}$ & $45 \mathrm{~mm}$ & $\begin{array}{l}\text { CR (CR-Flex): } 46 \\
\text { cases, PS (LPS- } \\
\text { Flex): } 45 \text { cases }\end{array}$ & Not described & Not described \\
\hline
\end{tabular}

\section{Outcomes}

Eight cases were revised for early aseptic loosening of the tibial component. Several additional patients have concerning radiographic signs of pending failure. The mean time from primary TKR to revision TKR was 17 months (range, 9-31 months).

Three cases underwent revision. HSS score increased from a median value of 35 to 95 (range: 78-100). KSS score increased from a median value of 31 points in the Knee and 45 points in the Function score to 95 (range: 83-100) and 94 (range: 81-100), respectively. The survival rate at 5 years was $97.9 \%$ with a $95 \%$ confidence interval.

The mean survival rate per Kaplan-Meier analysis was $99.4 \%$ at 6.1 years and there was no implant-related revision. The mean postoperative outcomes were: knee range of motion, 134.3 degrees; HSS score, 92.7; femorotibial angle, 5.2 degrees valgus; tibial component alignment angle, 90.2 degrees; tibial component posterior inclination, 4.8 degrees; the percentage of cases with tibial component alignment angle of $90 \pm 3$ degrees, $96.1 \%$; the percentage of cases with the femorotibial angle of $6 \pm 3$ degrees valgus, $94.0 \%$. Radiolucent lines were observed in 20 cases $(12.0 \%)$.

Compared with cases using standard components (standard group), the rate of revision for tibial aseptic loosing was significantly higher in the Mini-keel group with 12 cases $(5.7 \%)$ and 4 cases in the standard group (1.6\%) (P $=0.036)$. The use of Mini-keel appears to be a prognostic factor for surgical revision (hazard ratio $=3.86(1.23-$ 11.88), $\mathrm{P}=0.02)$ but not for the development of a radiolucent line $(\mathrm{HR}=1.75(0.9-3.4), \mathrm{P}=0.097)$.

The average postoperative outcomes were: knee range of motion, 127.8 degrees; HSS score, 90.6; KSS Knee score, 92.5; KSS Function score, 76.7; femorotibial angle, 5.0 degrees valgus; tibial component alignment angle, 90.2 degrees; tibial component posterior inclination, 4.6 degrees; the percentage of cases with tibial component alignment angle of $90 \pm 3$ degrees, $96.5 \%$; the percentage of cases with the femorotibial angle of $6 \pm 3$ degrees valgus, $83.6 \%$

The average postoperative outcomes were: knee range of motion, 133.4 degrees; KSS Knee score, 91.2; femorotibial angle, 5.7 degrees valgus; tibial component alignment angle, 90.5 degrees; the percentage of cases with tibial component alignment angle of $90 \pm 3$ degrees, $95.6 \%$; the percentage of cases with femorotibial angle of $6 \pm 3$ degrees valgus, $93.5 \%$.

KSS Function score at 5-year follow-up averaged 81.67 and 80.12 in the CR and PS groups, respectively $(p=0.29)$. The femorotibial angle averaged 5.85 degrees and 5.85 degrees valgus in the CR and PS groups, respectively $(\mathrm{p}=0.60)$. The average tibial component angle was 0.46 degrees and 0.61 degrees varus in the CR and PS groups, respectively $(\mathrm{p}=0.30)$. The average posterior inclination averaged 2.28 degrees and 1.93 degrees in the CR and PS groups, respectively $(p=0.51)$. Radiolucency was noted in 17 zones of the CR group and in 9 zones of the PS group $(p=0.24)$. Three TKRs required further surgery: one locking plate fixation for a periprosthetic tibial fracture (PS group) and two revision TKRs (one CR infection and one PS fracture). The mean survival rate at 5.7 years was $97.8 \%$ with a $95 \%$ confidence interval.
Notes

Intraoperatively, in all revised cases, more than $50 \%$ of the tibial tray was devoid of cement and factory-applied polymethylmethacrylate.

The mean delay before revision was 38 months (range: 8-64 months) in the Mini-keel group and 15.2 months (range: 8-22 months) in the standard group $(\mathrm{P}=0.006)$. Gender, $\mathrm{BMI}$ and pre- or postoperative alignment were not prognostic factors for revision or radiolucent lines. Navitrack-Orthosoft navigation system was used in all the cases.

A rupture of the medial collateral ligament occurred in two cases. The accuracy of the tibial implant alignment was slightly reduced in severe cases of preoperative varus deformity. Radiological outcomes and short-term clinical results were satisfactory regardless of the preoperative degree of varus deformity.

One case of rupture of the medial collateral ligament and one case of detachment of the cortex from the medial femoral condyle occurred. Compared to non-obese patients, obese patients showed satisfactory clinical and radiological results.

The overall complication rate was $6.7 \%$ in the CR group and $8.7 \%$ in the PS group. No significant difference was identified. Mini-keel showed good reliability and results with both CR and PS prostheses. 


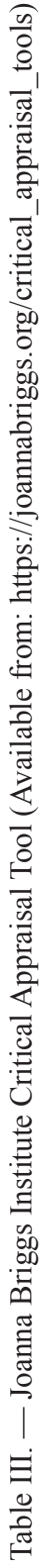
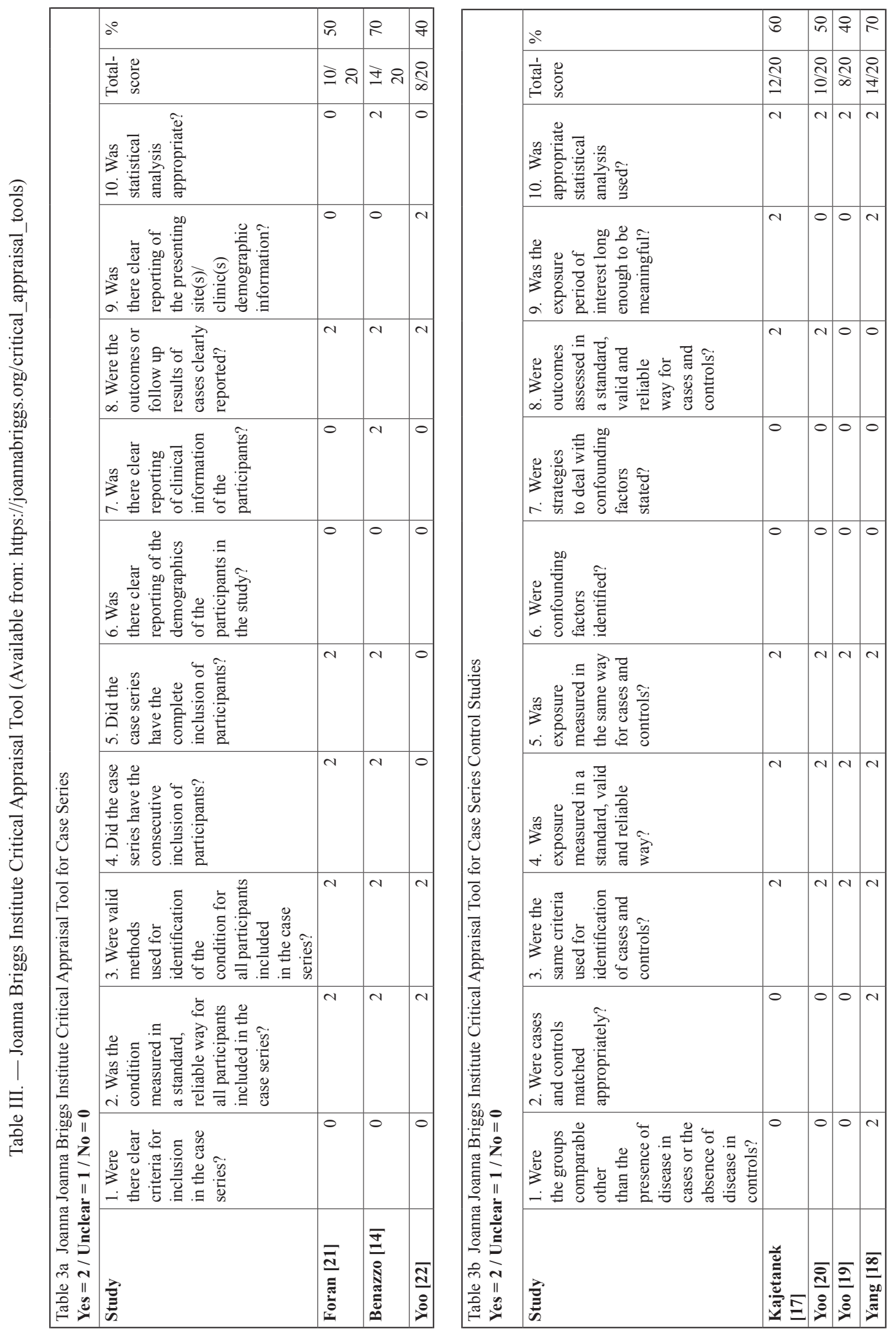
1 case of detachment of the cortex from the medial femoral condyle, and 1 case of periprosthetic tibial fracture) were reported in total. These complications may occur in MIS-TKR regardless of the use of a Mini-keel, as they might happen by excessive tension on the adjacent tissue when the tibial component was inserted with the knee extended. Mini-keel is designed to facilitate insertion of prostheses in the smaller surgical fields, however, care still should be taken to prevent tension on the surrounding tissue when it is inserted.

This systematic review found only one retrospective study which compared outcomes following TKR with the use of a Mini-keel and that with the use of a standard keel component, and no prospective (cohort) study or randomised-controlled trial was found. Kajetanek et al performed a casecontrol study in 459 consecutive TKRs (212 Minikeel and 247 standard components) and analysed the rate of revision for aseptic tibial loosening and identification of a radiolucent line after a median follow-up of 5 years. As a result, the rate of revision for tibial aseptic loosing was significantly higher in the Mini-keel group with 12 cases $(5.7 \%)$ and 4 cases in the standard group $(1.6 \%)(\mathrm{P}=0.036)$, while the decision to perform surgical revision was subjective, based on the patient's symptoms, X-ray interpretation, and the surgeon's experience. The use of a Mini-keel appeared to be a prognostic factor for surgical revision (hazard ratio $(\mathrm{HR})=3.86(1.23$ 11.88), $\mathrm{P}=0.02$ ) but not for the development of a radiolucent line $(\mathrm{HR}=1.75(0.9-3.4), \mathrm{P}=0.097)$. They concluded that the use of a Mini-keel was associated with a greater risk of revision for tibial component loosening. Note that the choice of the prosthesis was decided by the availability of components in the operating room and not by randomisation, which can be a limitation of this study.

Yoo et al. (19) showed MIS-TKR with the use of a Mini-keel in obese patients showed satisfactory clinical and radiological results. Yang et al. (18) described MIS-TKR with the use of a Mini-keel with both CR and PS prostheses showed good reliability. Yoo et al. (20) showed that satisfactory component alignment was achieved in MIS-TKR with the use of a Mini-keel regardless of the degree of varus deformity.
The strengths of this systematic review include the pursuit of knowledge in a new and important arena that has scarce published information and remains a topical subject for knee surgeons. The methodology is sound and encompasses a broadbased and comprehensive systematic literature search of multiple databases with multiple reviewers allowed for a very inclusive approach to capturing the vast majority of existing literature. In addition, the included studies were critically appraised using a validated quality measurement tool (JBICAC). Nonetheless, there are limitations which include the inclusion of English only studies and the overall low level of evidence available in the included studies on this topic. Non-prospective designs are prone to data inaccuracy as well as missing information, which subjects them to selection and source bias. Publication bias should also be recognised, and they may diminish the accuracy of the data collected and therefore limits the quality of a systematic review without a doubt.

\section{CONCLUSION}

Although over half of the studies found from this systematic review reported good and satisfying outcomes following MIS-TKR with the use of a Mini-keel, there still have been controversies among studies on this subject. There, unfortunately, remains a paucity of prospective comparative studies, which makes it difficult to perform a meaningful assessment of the use of a Mini-keel for MIS-TKR. To make any specific recommendations for knee surgeons with regard to the use of this implant for MIS-TKR, adequately powered longterm prospective cohort studies comparing MISTKRs with the use of a Mini-keel and those with the use of a conventional implant need to be conducted in the future.

\section{REFERENCES}

1. Argenson JN, Boisgard S, Parratte S, et al. Survival analysis of total knee arthroplasty at a minimum 10 years' follow-up : a multicenter French nationwide study including 846 cases. Orthop Traumatol Surg Res. 2013 Jun ; 99(4) : 385-390. 
2. Jauregui JJ, Cherian JJ, Pierce TP, Beaver WB, Issa K, Mont MA. Long-Term Survivorship and Clinical Outcomes Following Total Knee Arthroplasty. J Arthroplasty. 2015 Dec ; 30(12) : 2164-2166.

3. Victor J, Ghijselings S, Tajdar F, et al. Total knee arthroplasty at 15-17 years: does implant design affect outcome? Int Orthop. 2014 Feb ; 38(2) : 235-241.

4. Ruiz D Jr, Koenig L, Dall TM, et al. The direct and indirect costs to society of treatment for end-stage knee osteoarthritis. J Bone Joint Surg Am. 2013 Aug ; 95(16) : 1473-1480.

5. Tasker A, Hassaballa M, Murray J, et al. Minimally invasive total knee arthroplasty; a pragmatic randomised controlled trial reporting outcomes up to 2 year follow up. Knee. 2014 Jan ; 21(1) : 189-193.

6. Bozic KJ, Beringer D. Economic considerations in minimally invasive total joint arthroplasty. Clin Orthop Relat Res. 2007 Oct ; $463: 20-25$.

7. Tria AJ, Jr. Advancements in minimally invasive total knee arthroplasty. Orthopedics. 2003 Aug; 26(8 Suppl) : s859-863.

8. Tria AJ, Jr., Coon TM. Minimal incision total knee arthroplasty: early experience. Clin Orthop Relat Res. 2003 Nov ; 416 :185-190.

9. Cheng T, Liu T, Zhang G, Peng X, Zhang X. Does minimally invasive surgery improve short-term recovery in total knee arthroplasty? Clin Orthop Relat Res. 2010 Jun ; 468(6) : 1635-1648.

10. King J, Stamper DL, Schaad DC, Leopold SS. Minimally invasive total knee arthroplasty compared with traditional total knee arthroplasty. Assessment of the learning curve and the postoperative recuperative period. J Bone Joint Surg Am. 2007 Jul ; 89(7) : 1497-1503.

11. King JC, Manner PA, Stamper DL, Schaad DC, Leopold SS. Is minimally invasive total knee arthroplasty associated with lower costs than traditional TKA? Clin Orthop Relat Res. 2011 Jun ; 469(6) : 1716-1720.

12. Dalury DF, Dennis DA. Mini-incision total knee arthroplasty can increase risk of component malalignment. Clin Orthop Relat Res. 2005 Nov ; 440 : 77-81.

13. Barrack RL, Barnes CL, Burnett RS, Miller D, Clohisy JC, Maloney WJ. Minimal incision surgery as a risk factor for early failure of total knee arthroplasty. J Arthroplasty. 2009 Jun ; 24(4) : 489-498.

14. Benazzo F, Rossi SM. Modular tibial plate for minimally invasive total knee arthroplasty. Knee Surg Sports Traumatol Arthrosc. 2012 Sep ; 20(9) : 1796-802.
15. Methley AM, Campbell S, Chew-Graham C, McNally R, Cheraghi-Sohi S. PICO, PICOS and SPIDER: a comparison study of specificity and sensitivity in three search tools for qualitative systematic reviews. BMC Health Serv Res. 2014 Nov ; 14 : 579.

16. Moher D, Liberati A, Tetzlaff J, Altman DG. Preferred reporting items for systematic reviews and meta-analyses : the PRISMA statement. PLoS Med. $2009 \mathrm{Jul} ; 6(7)$ : e1000097.

17. Kajetanek C, Bouyer B, Ollivier M, Boisrenoult P, Pujol N, Beaufils P. Mid-term survivorship of Mini-keel versus Standard keel in total knee replacements: Differences in the rate of revision for aseptic loosening. Orthop Traumatol Surg Res. 2016 Sep ; 102(5) : 611-617.

18. Yang CP, Hsu KY, Chang YH, Chan YS, Shih HN, Chen AC. Mid-term survivorship of cruciate-retaining versus posterior-stabilized total knee arthroplasty using modular mini-keel tibial implants. J Orthop Surg Res. 2018 Feb; $13(1): 35$.

19. Yoo JH, Oh HC, Park SH, Kim JK, Kim SH. Does Obesity Affect Clinical and Radiological Outcomes in Minimally Invasive Total Knee Arthroplasty? Minimum 5-Year Follow-up of Minimally Invasive TKA in Obese Patients. Clin Orthop Surg. 2018 Sep ; 10(3) : 315-321.

20. Yoo JH, Park SH, Han CD, Oh HC, Park JY, Choi SJ. Radiologic Outcomes According to Varus Deformity in Minimally Invasive Surgery Total Knee Arthroplasty. Yonsei Med J. 2016 Jan ; 57(1) : 225-231.

21. Foran JR, Whited BW, Sporer SM. Early aseptic loosening with a precoated low-profile tibial component : a case series. J Arthroplasty. 2011 Dec ; 26(8) : 1445-1450.

22. Yoo JH, Park BK, Han CD, Oh HC, Park SH. Minimum 5-year Follow-up Results of Minimally Invasive Total Knee Arthroplasty Using Mini-Keel Modular Tibial Implant. Knee Surg Relat Res. 2014 Sep ; 26(3) : 149-154.

23. Smith TO, King JJ, Hing CB. A meta-analysis of randomised controlled trials comparing the clinical and radiological outcomes following minimally invasive to conventional exposure for total knee arthroplasty. Knee. 2012 Jan ; 19(1) : 1-7.

24. Walker PS, Soudry M, Ewald FC, McVickar H. Control of cement penetration in total knee arthroplasty. Clin Orthop Relat Res. 1984 May ; 185 : 155-164. 